Research Article

\title{
Identification of Critical Links in Urban Road Network considering Cascading Failures
}

\author{
Yulin Tian $\mathbb{D}^{1},{ }^{1}$ Xiaoming Liu, ${ }^{1}$ Zhen ${ }^{2},{ }^{2}$ Shaohu Tang, ${ }^{3}$ Chunlin Shang, ${ }^{1}$ and Lu Wei ${ }^{1}$ \\ ${ }^{1}$ Beijing Key Lab of Urban Road Traffic Intelligent Tech., North China University of Technology, Beijing 100043, China \\ ${ }^{2}$ China Software Tesing Center, Beijing 100048, China \\ ${ }^{3}$ College of Urban Rail Transit and Logistics, Beijing Union University, Beijing 100101, China
}

Correspondence should be addressed to Yulin Tian; tian_yulin@yeah.net

Received 17 March 2021; Revised 9 April 2021; Accepted 13 April 2021; Published 24 April 2021

Academic Editor: Mohammad R. Khosravi

Copyright (c) 2021 Yulin Tian et al. This is an open access article distributed under the Creative Commons Attribution License, which permits unrestricted use, distribution, and reproduction in any medium, provided the original work is properly cited.

\begin{abstract}
The identification of critical road links is greatly important to the management and control of the transportation system. Existing works fail to fully consider the influence of the distribution of traffic flow and its dynamic characteristics on critical road link identification. In this paper, we propose a criticality calculation method for urban road networks considering the effect of cascading failures which models the distribution change of traffic flow after a specific road link failed. Firstly, a sequence diagram calculation method is proposed to model how the traffic failure on one road link propagates to related links. Secondly, the diagram of the cascade failure sequence is divided into different stages according to the consistency of the objective function. The influence value of each stage is computed for the target road link. Finally, the failure probability model and the importance indicator are proposed to calculate the criticality for each road link. We evaluate our critical road link identification method on both simulated and read scenes. In our simulation, we achieve $90.6 \%$ and $91.7 \%$ for the accuracy on two key metrics, respectively, i.e., the length of failure road link and the total parking delay, which proves the feasibility of our method. Our method also achieves reasonable conclusions on real data and helps to find the critical road links.
\end{abstract}

\section{Introduction}

In recent years, with the gradual acceleration of urbanization, the demand for transportation resources has increased rapidly, which has led to the rising pressure on urban road traffic and the increasingly serious traffic congestion problem. Urban traffic congestion often occurs in some specific locations or areas. Although the number of these critical road links is small, they can directly or indirectly affect the status of the surrounding regional road network; moreover, they are one of the important reasons for congestion in the regional road network. The effective identification of critical road links plays a very important role in formulating traffic management measures, alleviating traffic congestion, and improving the travel quality of residents.

This paper is based on the viewpoint proposed by Taylor et al. [1]: the so-called critical road links should have a high degree of vulnerability, that is, a higher probability of failure and a high degree of importance; that is, the failure has a more serious impact on the road network. Currently, the vulnerability of critical road links is generally determined by the probability of being failed [2-4]; the calculation of importance is more complicated, and it is also a key part of critical road links determination $[5,6]$. The current researches in this area could be divided into two aspects, that is, the impact of a road link failure on the structure and operation efficiency of the road network.

In terms of the impact of a road link failure on road network structure, some researchers adopted complex network theory to traffic networks. Sanchez et al. [7] proposed a resource allocation model based on the operation reliability of traffic road networks, which could identify critical road links in the road network by calculating the impact of road links on the accessibility of the network. Este et al. [8-10] identified critical road links by the changes of road network connectivity, road link betweenness, and road 
network robustness before and after a road link failure. Tu et al. [11] proposed a method for calculating the criticality of road links based on the minimum cut frequency vector indicator, which identifies critical road links from the perspective of road network topological vulnerability. Ball et al. [12] used the change in the shortest path of the network after removing a road link in the network to determine its importance. Girvan et al. [13] proposed the concept of edge betweenness to measure the importance of edges in a network based on betweenness centrality. The larger the edge betweenness, the more important the edge is in the network and the stronger the transmission ability and control ability of the edge to network resources. Lu et al. [14] used the entropy weight method to evaluate the three edge importance indicators: the number of edge betweenness, the decline rate of spanning tree number, and the growth rate of average distance, and then obtained the comprehensive weight of the three indicators to rank the edge importance. There are also some studies that identify critical road links in road networks based on complex network indicators such as degree, k-core decomposition, and proximity after pairwise modeling of urban traffic road networks $[15,16]$. Most of the studies in this area are based on complex network technology and use structure-based key point identification methods in complex networks to identify critical road links in road networks, and these studies often lack in-depth research on traffic flow in the process of identifying critical road links in road networks.

In terms of the impact of a road link failure on the operational efficiency of the road network, researchers tend to adopt the traffic assignment theory to calculate the impact of road link failure and measure the impact of the road link on the entire network by the changes in the identification indicators before and after the road link failure. Jenalins et al. [17] identified critical road links in the road network from the consequences of road link failure, which used a user equilibrium model to allocate traffic flows before and after a road link failure, and determine the importance of each road link by comparing the travel costs in the road network before and after failure. Scott et al. [18, 19] established a network robustness index to measure the criticality of road links by the change in vehicle travel time after a road link failure. Zhang et al. [20] considered the impact of a road link failure on the local road network, optimized the traffic network reconstruction model within the area affected by the road link failure, and combined the changes of traveler time cost indicator and the local road network traffic load indicator to calculate the road link importance. Researchers have studied the importance of road links not only from the perspective of the total travel cost of the transportation system but also from the diversity of individual travel choices and individual travel efficiency. To evaluate the effect of a road link failure on route choice and network operation efficiency in a traffic network, a sort of road link importance ranking method is proposed, which takes the route redundancy index and travel efficiency index into consideration [21]. The paper comprehensively evaluated the importance of road links by the change degree index of feasible and reasonable number of routes individual travel time consumption before and after link failure. Since these studies require the calculation of the impact after road link failure (road link failure is under a hypothetical scenario), which also requires the reallocation of traffic flows on the road network, the value of the impact before and after road link failure depends to a large extent on the accuracy of the traffic flow allocation. In the actual road network, the traffic flow redistribution process is difficult to describe precisely due to the influence of intersection traffic signal schemes and the random choice of travelers, resulting in an overly ideal process for road link failure impact assessment by this type of method, which is not effective in practice.

Based on the current study, this paper starts from the process of cascade failure of road links under the action of traffic flow and studies the real-time impact on the road network after the failure of the road link. The road link failure studied in this paper is mainly due to the inability of the road link traffic flow to evacuate in time, resulting in a sharp reduction in the actual capacity of the road link and a temporary loss of function that allows vehicles to pass. Road link failures are usually followed by overflow, which then spreads to upstream intersections, causing deadlocks in each entrance at upstream intersections, and then road links continue to overflow, resulting in cascade failures.

In this paper, we propose a model for calculating the criticality of road links considering the cascade failure to evaluate the degree of impact on the road network after a road link failure. We use the dynamic propagation characteristics of traffic flow and combine with traffic wave theory to model the failure process of road links and then use the distribution of traffic flow in the road network and the road network's topology to achieve the description of the cascade failures. We start from the mathematical description of the traffic overflow caused by road link failure, establish a road link queue spreading model, construct a time sequence diagram of road link cascading failure by combining the road network topology and the time required for a complete failure of a road link, calculate the failure impact indicators by piecewise solution to obtain the total impact value of section failure, and finally combine the road link importance indicator with the road link failure probability indicator based on observation and statistical methods to complete the calculation of road link criticality. The contributions of our work are summarized as follows: we propose a method for evaluating the importance of road links considering cascading failures, which improves the reliability and practicality of existing methods; we propose a method of time sequence diagram, which solves the problem of calculating the impact of road links cascade failures. We also propose a vehicles overflow model based on the uniform arrival of traffic flow and traffic flow theory, which improves the impact of traffic signal control schemes on traffic flow modeling and lays the foundation for establishing an accurate failure impact model.

\section{Materials and Methods}

2.1. Identification Model for Critical Links. The criticality of the road link is positively related to its vulnerability and 
importance [19]. The weaker the stability and the greater the impact after failure, the higher the criticality of the road link. In this paper, we use the product of vulnerability and importance indicators to represent the criticality of the road link, the model for calculating the criticality of a road link is described as follows:

$$
C_{a}=P_{a} \cdot K_{a}
$$

where $C_{a}$ is the criticality of road link $a, P_{a}$ and $K_{a}$ are the value of vulnerability and importance indicators for road link $a$.

2.2. Modeling for Vulnerability of Road Link. The vulnerability of the road link is calculated by its failure probability. We choose the speed and saturation criterion [21], combined with the discriminatory rules on the service level of road link in Highway Capacity Manual 2000 (USA), as the basis for discriminating failure status of a road link. The failure probability of a road link is defined as the ratio of the sum of the dispersed time periods in which the road link is in a failed state to the length of the continuously observed time period. The mathematical expression is shown below:

$$
P_{a}=\frac{t_{T}^{f}}{T}=\frac{t_{1}^{f}+t_{2}^{f}+\cdots+t_{n}^{f}}{T},
$$

where $t_{T}^{f}$ is the total time that the road link is in the failure state during the observation time, $T$ is the total observation time, and $t_{i}^{f}$ is the duration of the $i$ th failure state.

2.3. Modeling for Importance of Road Link. In this paper, the importance of road link is modeled in terms of their actual impact on the road network under the action of traffic flow after road link failure. When a road link fails, it will cause a cascading failure response to the surrounding related road network. To model the impact level of this process in real time, we need to determine the failure time sequence of the related road links, that is, the moment and duration of the failure and its influence. The computational effort of this process rises rapidly with the proliferation of cascading failures in the road network, making it more complex to be described directly mathematically. Instead, we propose a method to calculate the importance of road links by constructing time sequence diagrams for cascade failures. The method firstly calculates the time when each road link starts to become failed and the time when it becomes completely failed, then carries out segmentation calculation by combining the failure influence function of each road link and finally realizes the real-time description of the cascade failure process of the road link to make up for the shortcomings of direct calculation. Without loss of generality, we first make the following assumptions.

Assumption 1, the road link failure studied in this paper mainly refers to a phenomenon that the actual capacity of the road link is drastically reduced due to the unbalanced traffic signal control scheme or excessive traffic saturation. When the target road link fails, we approximate that the actual capacity at the exit on the section is reduced to 0 for a certain period of time so as to simulate the dynamic impact process of traffic flow on the road network under this scenario.

Assumption 2, considering that the starting point of road link failure has a certain influence on the cascade failures on related road links, in order to facilitate the mathematical description of the failure process, we approximate the failure starting point to be the edge point of the road link.

Assumption 3, in the process of cascade failure, considering that the traffic signal scheme affects the arrival distribution of traffic flow on the road links and the failure sequence of different entrance road links in the intersection, which in turn increases the difficulty of building the traffic flow arrival model, we use the average value of the traffic flow to describe the traffic flow arrival distribution on each road link.

\subsubsection{Time Sequence Diagram of Road Link Cascading} Failure. $N, E$ are the sets of nodes (intersections) and edges (road links) in the road network $G=(N, E)$, respectively. $e_{i j}$ indicates the traffic flow from intersection $n_{i}$ to intersection $n_{j} . t_{j i}$ indicates the time required for queuing vehicles to overflow from intersection $n_{j}$ to intersection $n_{i}$ after the road link $e_{i j}$ fails. $l_{i j}$ indicates the length of the corresponding road link. $q_{i j}$ is the hourly average traffic volume of the road link $e_{i j}$. The time sequence diagram of road link cascading failure (as Figure 1) is generated as follows.

Step 1. Get the input values of the algorithm: the road link $e_{i j}$ and its initial spreading direction $\underset{e_{j i}}{\longrightarrow}$.

Step 2. Set the target road link parameters: average flow $q_{i j}$, jam density $k_{j}^{i j}$, free-flow speed $u_{f}^{i j}$.

Step 3. Calculate the stop-wave speed $u_{w}^{i j}$ of the target road link $e_{i j}$ in the initial spreading direction.

Step 4. Calculate the time required for the target road link to reach the complete failure state and then plot the corresponding failure time segments.

Step 5. Get the set of road links that will fail at the next level after each road link spreads to the intersection and execute Step 2 to Step 5 for each section one by one.

2.3.2. Evaluation Indicators for the Impact of Road Link Cascading Failure. The impact of a road link failure on the road network is multifaceted. From the perspective of driver travel costs, the vehicles affected by a road link cascade failure could not move normally, the number of vehicles affected is the direct impact of a road link failure, and the total parking delay is the direct cost of the impact of a road link failure. Analyzed from the perspective of road network service capability, after the failure of the road link, the length of the available road link in the road network is reduced, which will reduce the service capability of the road network under the condition that the total amount of road network resources remains unchanged, and the length of the failed 


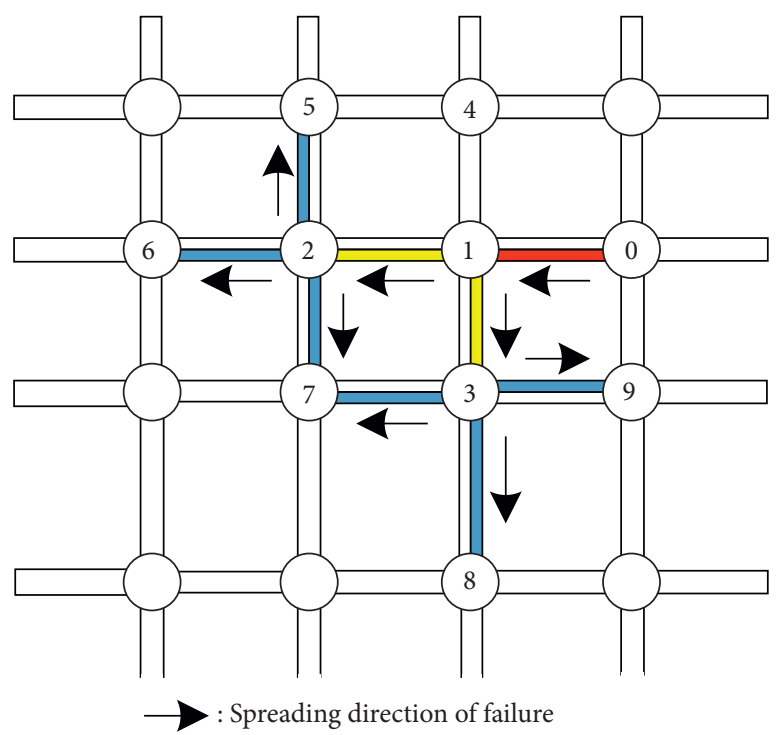

(a)

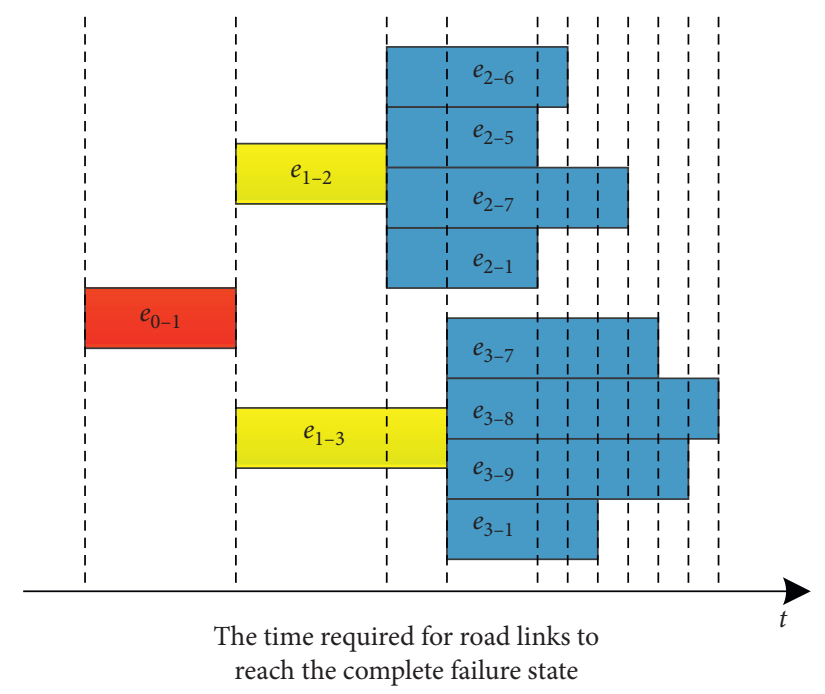

(b)

FIGURE 1: Time sequence diagram of road link cascading failure. (a) Description of cascading failures in a road network. (b) Description of a corresponding time sequence diagram.

road link is the indirect cost of the impact of road link failure. From the relationship between saturation and operation efficiency in the road network [22], it can be seen that the parking delay indicator is suitable for evaluating the operational efficiency when the road network saturation is low, while the failure road links length indicator is more suitable when the road network saturation is high. We adopt these two parameters as the evaluation indicators for the impact of road link failure.

Considering the time-varying characteristics of road network saturation, we assign different weights to two evaluation indicators to get the impact value of road links failures under different saturation.

$$
k_{m n}^{t_{i}, t_{i+1}}=\propto_{1} T_{m n}^{t_{i}, t_{i+1}}+\propto_{2} L_{m n}^{t_{i}, t_{i+1}},
$$

where $k_{m n}^{t_{i}, t_{i+1}}$ is the failure impact value of road link $e_{m n}$ in time period $\left(t_{i}, t_{i+1}\right), T_{m n}^{t_{i}, t_{i+1}}$ and $L_{m n}^{t_{i}, t_{i+1}}$ are the corresponding parking delay and failure road links length, $\alpha_{1}, \alpha_{2}$ are the weight coefficients of the two indicators to achieve an objective description of road link failure impact.

\subsubsection{Piecewise Solution Based on Time Sequence Diagram of} Road Link Cascading Failure. After the construction of time sequence diagram of road link cascading failure, we need to segment the time sequence diagram to ensure that the failure impact functions within each segment are the same, which facilitates the establishment of a complete time-varying model considering the impact of road link cascading failure. In the process of piecewise solution, we first need to slice the time sequence diagram of road link cascading failure according to the start and stop moments of each new road link, and then solve the failure impact function of each road link within the segment and establish the impact calculation model after the failure for the target road link by summing the impact values of each segment.

$$
K(t)=\sum k_{m n}^{t_{i}, t_{i+1}}, \quad t_{i} \in(0, t), t_{i+1} \in(0, t) .
$$

2.4. The Process of Single Road Link Failure. After the failure of a road link, the vehicles arriving one after another will generate queues and the consequential stop-wave of queuing vehicles will gradually move backward. We establish the dynamic queuing model (as Figure 2) after the failure of a road link based on the traffic wave theory. A traffic wave is a phenomenon representing the speed fluctuation of the traffic flow, which is caused by the density change of traffic flow. It can be divided into rarefaction-wave and queuing-wave according to the distribution of vehicle density in the moving direction of traffic flow. The speed of the wave is mainly affected by the input traffic volume of the road link and the spatial distribution of vehicles.

In the process of road link failure, vehicles arrive at the failure point at a certain speed and form a queue, which will increase the density of traffic flow. When the traffic wave propagates from the low-density area to the high-density area, the traffic wave can be modeled as follows:

$$
\left(u_{1}-u_{w}\right) \cdot k_{1}=\left(u_{2}-u_{w}\right) \cdot k_{2} \text {. }
$$

From the relation between the average speed $u_{i}$ of traffic flow on the road link and the vehicle density $k_{i}$, we transform the propagation equation of traffic wave as follows:

$$
u_{w}=\frac{\left[k_{1} u_{f}\left(1-k_{1} / k_{j}\right)\right]-\left[k_{2} u_{f}\left(1-k_{2} / k_{j}\right)\right]}{k_{1}-k_{2}} \text {. }
$$




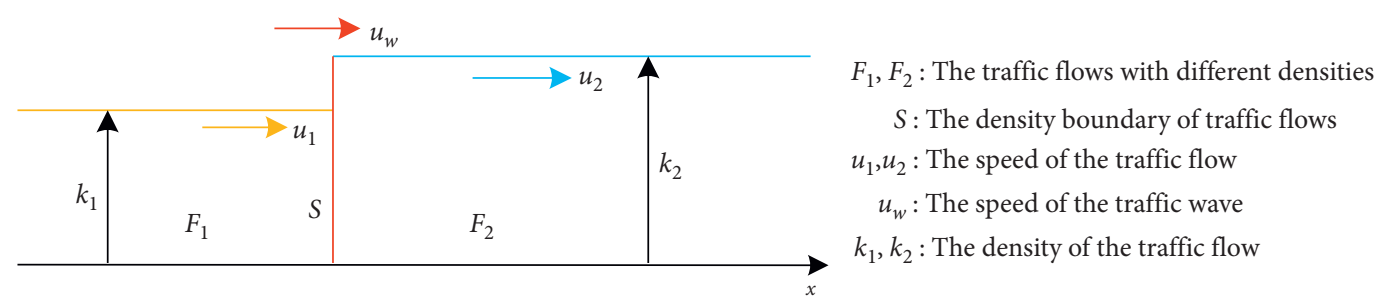

Figure 2: Operation diagram of traffic flow with different density.

When the road link fails, the traffic flow in the highdensity area is in the stopped state. That is $k_{2} / k_{j}=1$, the overflow speed now is as follows:

$$
u_{w}=-\frac{u_{f}}{k_{j}} \cdot k_{1} .
$$

We convert the overflow speed according to the relation between the average traffic flow $q$ and the average speed $u$ in road links. That is $q=k_{j}\left(u-u^{2} / u_{f}\right)$, the stop-wave speed $u_{w}$ can be expressed by the average traffic flow $q$ as follows:

$$
u_{w}=\left(\frac{u_{f}^{2}}{4}-\frac{u_{f}}{k_{j}} q\right)^{(1 / 2)}-\frac{u_{f}}{2} .
$$

As can be seen from the above equation, after the road link failure, the overflow speed $u_{w}$ is mainly affected by the real-time traffic flow $q$. We can get the overflow speed value of each road link by obtaining the traffic flow values, combined with the basic parameters $k_{j}, u_{f}$ when the vehicles are running on the road links.

2.5. The Process of Cascading Failure on Multiple Road Links. When the road link failures spread from a single link to multiple links, the impact of the failures on the road network is no longer a simple sum of the impact values of each link but should also consider the mutual impact of each road link.

\subsubsection{Sequential Failure Process of Upstream and Down-} stream Intersections. When the cascade failure propagates from intersection $n_{i}$ to $n_{j}$, the road link failure continues to spread along the entrance lanes of intersection $n_{j}$, but since the upstream intersection $n_{i}$ has already failed, it no longer transmits traffic flow to intersection $n_{j}$, so these road links, which are connected to the upstream intersections, no longer generate overflow when upstream and downstream intersections fail sequentially. The status function to show whether a road link will overflow is as follows:

$$
\begin{cases}f_{s}^{i j}=0, & n_{i} \in N_{\mathrm{fld}}, \\ f_{s}^{i j}=1, & n_{i} \in N_{\mathrm{nrm}},\end{cases}
$$

where $N_{\text {fld }}, N_{\text {nrm }}$ are the sets of failed and normal intersections respectively; $f_{s}^{i j}$ is the state factor in the cascade failure process, and a value of 0 indicates that the road link is failed, while a value of 1 indicates that the road link is normal. The scenario is shown in Figure 3.
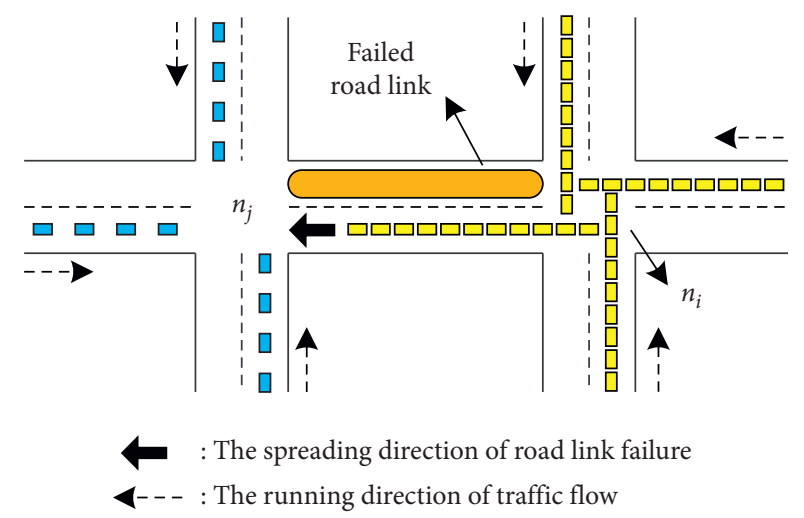

FIgURE 3: Diagram of upstream and downstream intersections failed successively.

2.5.2. Failure Spreading Process of Upstream Intersection. When the traffic overflow caused by road link failure propagates to the intersection, the road link failures phenomenon will spread along each entrance lane in the intersection (as Figure 4), and the impact of the road link failure in this process is the sum of the real-time impact of each road link.

Combining the process of sequential failures at upstream and downstream intersections, we can calculate the real-time number of affected vehicles when the road link failure spreads from the intersection, as shown below.

$$
N(t)=\sum_{j} f_{s}^{i j} \frac{u_{w}^{i j} \cdot t}{d_{v h}^{i j}}, \quad v_{j} \in V_{\mathrm{des}},
$$

where $V_{\text {des }}$ is the set of intersections that are the first-order neighbors of current failed intersection, $d_{v h}^{i j}$ is the distance of headway for cars on road link $e_{i j}$. The time-varying function of the total delay of the affected vehicles is as follows:

$$
T(t)=\sum_{j} \int f_{s}^{i j} \frac{u_{w}^{i j} \cdot t}{d_{v h}^{i j}} \mathrm{~d}_{t}, \quad v_{j} \in V_{\mathrm{des}} .
$$

The time-varying function of failure road links length is as follows:

$$
L(t)=\sum_{j} f_{s}^{i j} \cdot u_{w}^{i j} \cdot t, \quad v_{j} \in V_{\mathrm{des}}
$$

2.5.3. Failure Arrival Process of Downstream Intersection. Among multiple upstream traffic overflows caused by road link failure, the one that spreads to the downstream 


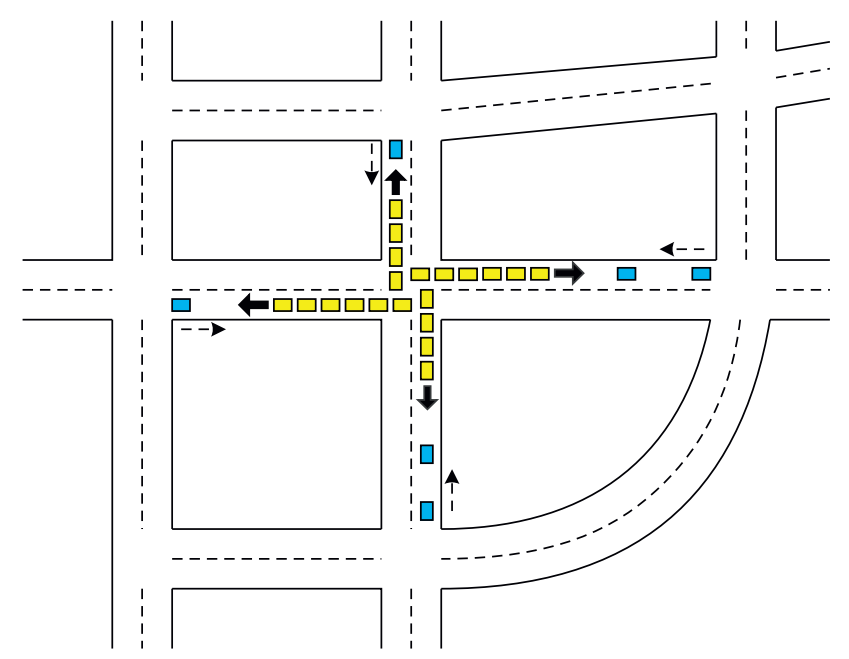

$\square-\rightarrow$ : The running direction of traffic flow

$\square \rightarrow$ : The spreading direction of road link failure

FIGURE 4: Diagram of road link failure starting from an intersection.

intersection firstly will gradually make the intersection fail and thus will stop the vehicles driving to other road links that are already overflowed (as Figure 5).

The number of affected vehicles $N(t)$ before and after the intersection failure moment $t_{\mathrm{mo}}$ is shown below.

$$
\begin{cases}N(t)=\sum_{m} f_{s}^{\mathrm{mo}} \cdot \frac{u_{w}^{\mathrm{mo}}}{d_{v h}^{\mathrm{m}}} t, & v_{m} \in V_{\mathrm{ori}}, t_{\mathrm{mo}}^{\prime}<t<t_{\mathrm{mo}}, \\ N(t)=\frac{l^{\mathrm{co}}}{d_{v h}^{\mathrm{co}}}+\sum_{n} f_{s}^{\mathrm{no}} \cdot f_{t}(n) \frac{u_{w}^{\mathrm{no}}}{d_{v h}^{\mathrm{no}}} t, & v_{n} \in V_{\text {ori }}, n \neq c, t_{\mathrm{mo}} \leq t \leq t_{\mathrm{no}}^{\prime},\end{cases}
$$

where $V_{\text {ori }}$ is the set of upstream intersections, $l^{c o}, d_{v h}^{\text {co }}$ are the length of road link $e_{\mathrm{co}}$ and the distance headway, respectively; $t_{\mathrm{mo}}^{\prime}$ is the starting moment when multiple road links are all overflowed, $t_{\mathrm{mo}}$ is the moment when the intersection fails, and $t_{\mathrm{no}}^{\prime}$ is the moment when all exit links of the intersection fail; $f_{t}(n)$ is the state factor of road links cascading failures, if it meets the condition that $t \geq t_{\mathrm{no}}^{\prime}$, the value will be 0 , which means that the road link is failed. Otherwise the road link is normal.

The corresponding total parking delay and failure road links length are as follows:

$$
\begin{aligned}
& T(t)=\int_{t s}^{t e} N(t) \mathrm{d} t, \\
& \begin{cases}L(t)=\sum_{m} f_{s}^{\mathrm{mo}} \cdot u_{w}^{\mathrm{mo}} \cdot t, & m \in V_{\mathrm{ori}}, t_{\mathrm{mo}}^{\prime}<t<t_{\mathrm{mo}}, \\
L(t)=\sum_{n} l^{\mathrm{no}}, & n \in V_{\mathrm{ori}}, t_{\mathrm{mo}} \leq t .\end{cases}
\end{aligned}
$$

2.6. Algorithm for Calculating the Criticality of Road Links. According to the cascade failure impact model and the criticality solution method, we design the following steps to

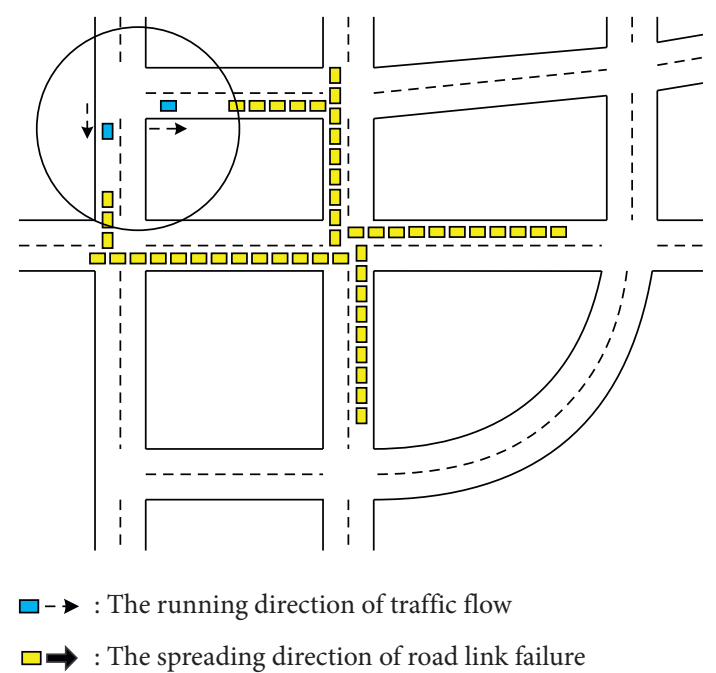

FIgURE 5: Diagram of multiple failed road links spreading downstream.

identify critical road links combined with the road link attributes and the traffic flow in the road network.

Step 1. Input the target road link $e_{i j}$ and topology parameters of the related road network.

Step 2. Calculate and draw the time sequence diagram of road link cascading failure.

Step 3. Divide the timing segments with the same failure effect function into one stage $S_{i}$.

Step 4. Calculate the dynamic impact value $N(t), T(t), L(t)$ of each stage.

Step 5. Sum up the impact values of each stage in the target time period and combine the weights of different failure impact indicators to get the importance indicator values $K_{a}$ of the target road link.

Step 6. Calculate the probability value $P_{a}$ of road link failure and compute the criticality value $C_{a}$ by combining the importance indicators value $K_{a}$ of road link.

\section{Results and Discussion}

To ensure the feasibility and accuracy of the method proposed in this paper that identification of critical links considering cascading failures, we first validate the proposed cascading failure impact model of road links to determine its feasibility, and then conduct experiments and analyze the critical road links identification method.

3.1. Validation of the Cascade Failure Impact Model. The cascade failure impact model is the core of the critical road link identification method in this paper. To ensure the feasibility of this calculation model, we conduct simulation experiments to verify the cascade failure impact model (as Figure 6), which uses real urban road networks and their corresponding traffic flow data.

We conduct simulation experiments with VISSIM, and the road network of Zhongguancun West District in Beijing 


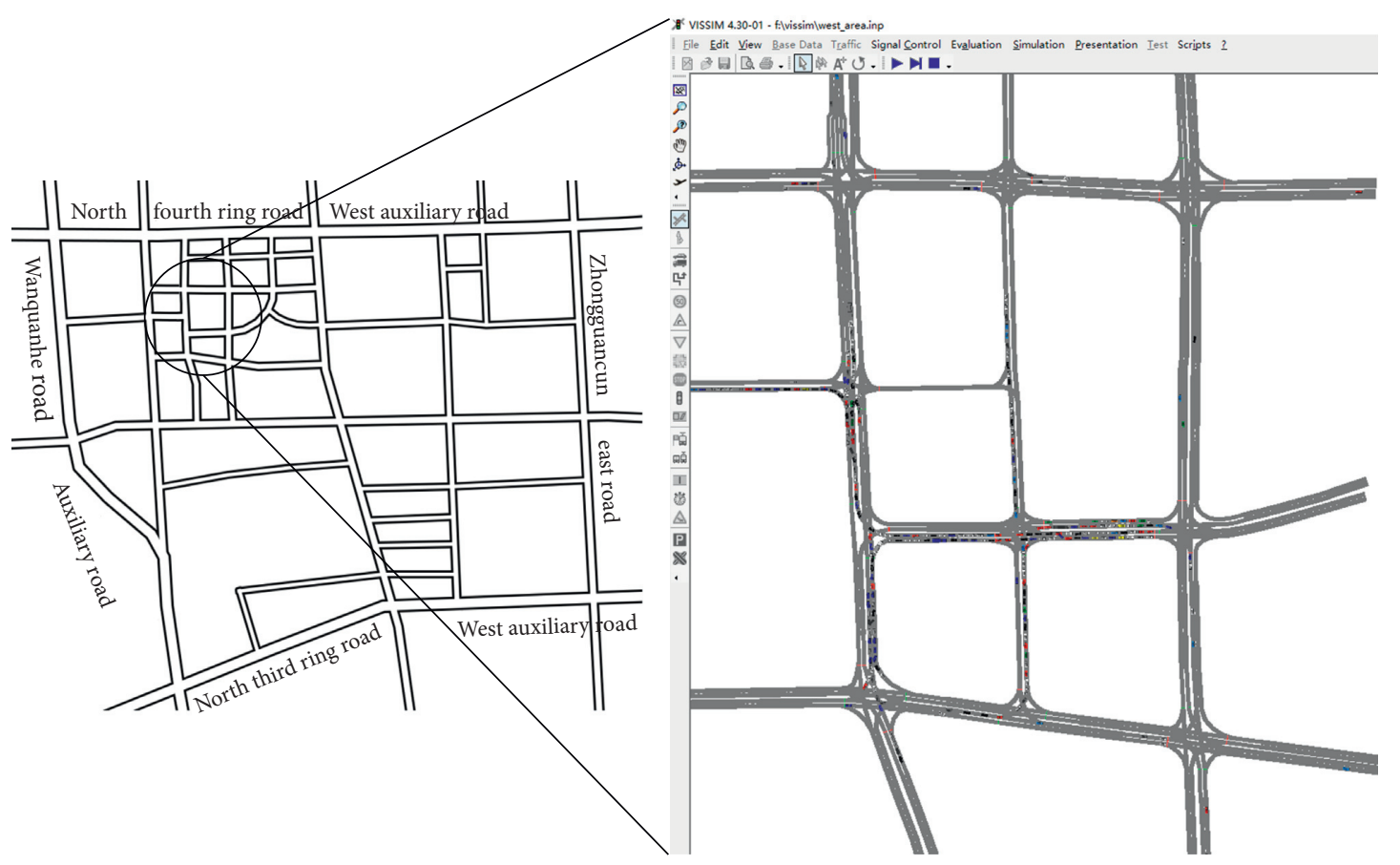

FIGURE 6: Simulation of road network cascading failure process.

is selected as the experimental area in this paper. To validate the cascade failure impact model quickly and efficiently, we select a part of the target road network for simulation experiments.

The data required in the experiment are divided into two parts, traffic flow data and road link basic data, where the basic data includes the number of lanes, channelization, length, failure probability, vehicle jam density, and free-flow speed of each road link. To ensure the accuracy of the experimental data, we used various ways to obtain the data. We built the basic data set of road links through the output interface of map software, manual survey, and field measurement. We counted, filtered, and analyzed the traffic flow of road links in the target area by video detectors and calculated the average hourly traffic volume of each road link according to different periods (where 09:30-12:00, 13: $30-16: 30$ are ordinary period; $07: 30-09: 30,16: 30-18: 30$ are peak period), and the traffic data set covers the period from September 7th to November 27th.

After the completion of the simulation environment and the selection of the target failure link, we count and output the values of three failure impact indicators: the total number of affected vehicles, the total parking delay, and the length of the failure road links, and compared them with the calculation results of the failure impact model to verify the feasibility of the proposed model. Figure 7 shows the comparison results.

To verify the actual effect of the cascade failure impact model under different traffic saturation, we select the ordinary period and peak period of the traffic and record the results under those two scenarios, that is, the model calculation and simulation experiments. By comparing the data result curves of two methods in three indicators, we can see that the cascade failure impact model proposed in this paper can be better fitted with the simulation results. The effectiveness and feasibility of this model can be verified by sampling the errors of each index under different time periods. The comparison results using Mean Absolute Percentage Error (MAPE) are shown in Table 1.

\subsection{Experiment and Analysis of Critical Section Identification} Methods. To verify the practical effect of the critical road links identification method considering cascading failures proposed in this paper, experiments are conducted with actual road links in the target area (about 6 square kilometers, as Figure 8), which includes 26 major road links. Through the actual investigation and measurement of the road network topology and road channelization, combined with the traffic flow data of the road links at different time periods, we build the experimental scenario of the target road network.

Combined with the analysis of this paper on the relations between road network saturation and importance evaluation indicator of road links, in the experiment, we adopted the total parking delay of affected vehicles as the evaluation indicator of importance (that is $\alpha_{1}=1, \alpha_{2}=0$ ) during the ordinary period and adopted the failure road links length as the evaluation indicator of importance (that is $\left.\alpha_{1}=0, \alpha_{2}=1\right)$ during the peak time. Considering that the cascade failures in the actual road network will not continuously spread, and the road link failure impact range increases sharply with time, we choose the 16th minute as the time node and rank the criticality of each road link based on the failure impact value at this time. We conducted experiments on the road network of a Wednesday. The 

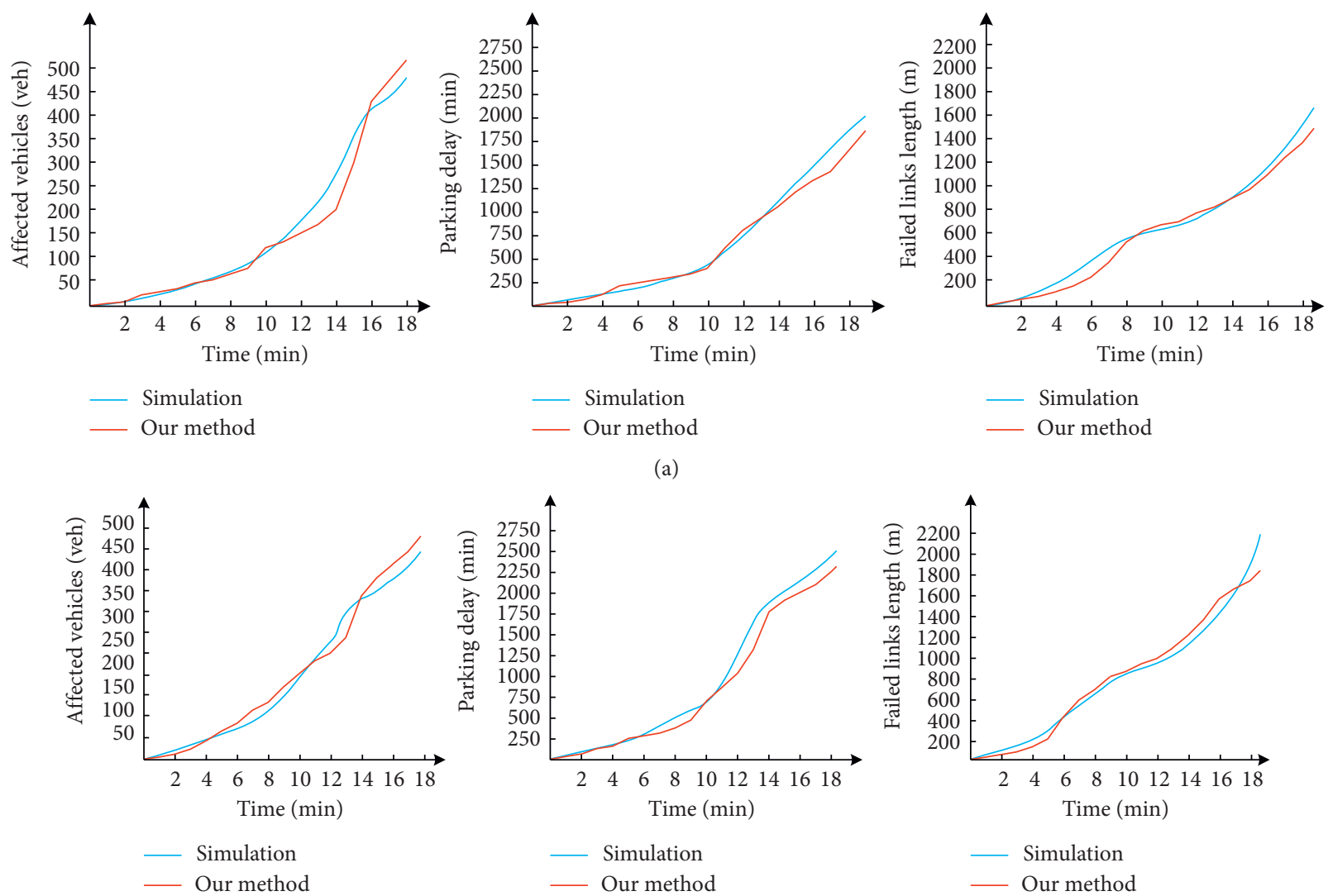

(b)

FIgURE 7: Comparison diagram of the failure impact model results. (a) Description of results during the ordinary period. (b) Description of results during the peak period.

TABle 1: The error rates of this method compared with simulation results.

\begin{tabular}{lccc}
\hline & $N(t)(\%)$ & $T(t)(\%)$ & $L(t)(\%)$ \\
\hline Ordinary period & 10.2 & 9.4 & 8.3 \\
Peak period & 10.6 & 10.1 & 9.3 \\
\hline
\end{tabular}

distribution of criticality of each road link is shown in Figure 9.

Through the calculation of the experimental data, we rank the criticality of different time periods and listed the road links with higher criticality in Tables 2 and 3.

The road links with high criticality under different time periods are listed in Tables 2 and 3. Through the analysis of the experiment results and combined with the specific conditions of the road links with high criticality, it can be seen that the critical links have the following characteristics.

(1) Not all critical road links in the road network are concentrated in the road links with high traffic flow or strong topological connectivity, and the road links with shorter length, fewer lanes, or adjacent to the entrances and exits of arterial roads may have a greater impact on the operational efficiency of the road network.

(2) When the road network is in the ordinary period, the criticality of arterial road links is higher. This is due to the uniform distribution of traffic flow and the low saturation of the road network. So, drivers tend to choose arterial road links with shorter travel time when traveling by car.

(3) When the road network is in the peak period, those bottleneck road links with fewer lanes or shorter link length have a higher criticality, which is due to the higher saturation of traffic flow on the road network, while bottleneck road links with weaker stability are more likely to fail first, which then quickly spread to the surrounding related road links and are more likely to form the cascade failures.

According to the above method to identify critical road links of the actual road network, it can be seen from the results that during the ordinary hours, the critical road links identified by this method are mostly distributed in the road links with high traffic flow such as the entrances and exits of arterial roads, while during the peak hours, the critical road links identified are mostly distributed in the road links with 


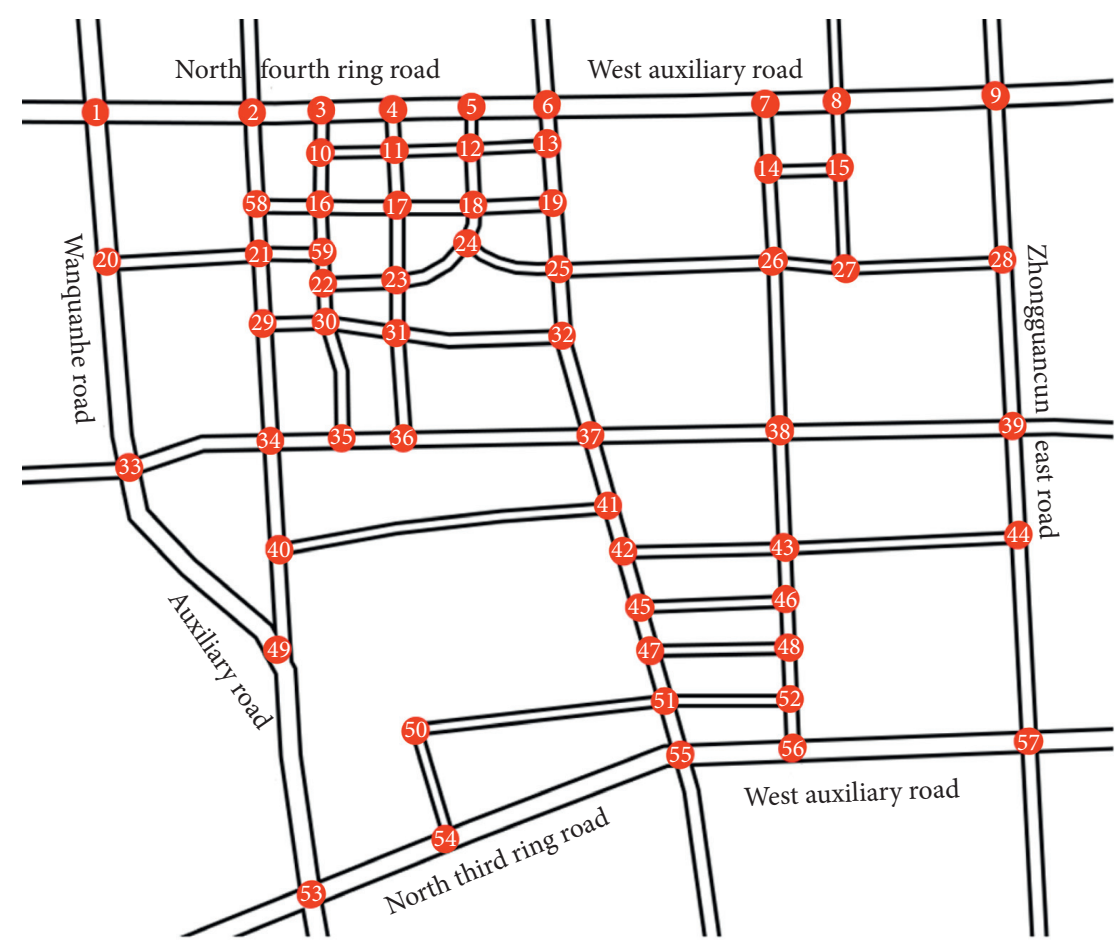

FiguRe 8: Road network and topology of the experimental area.

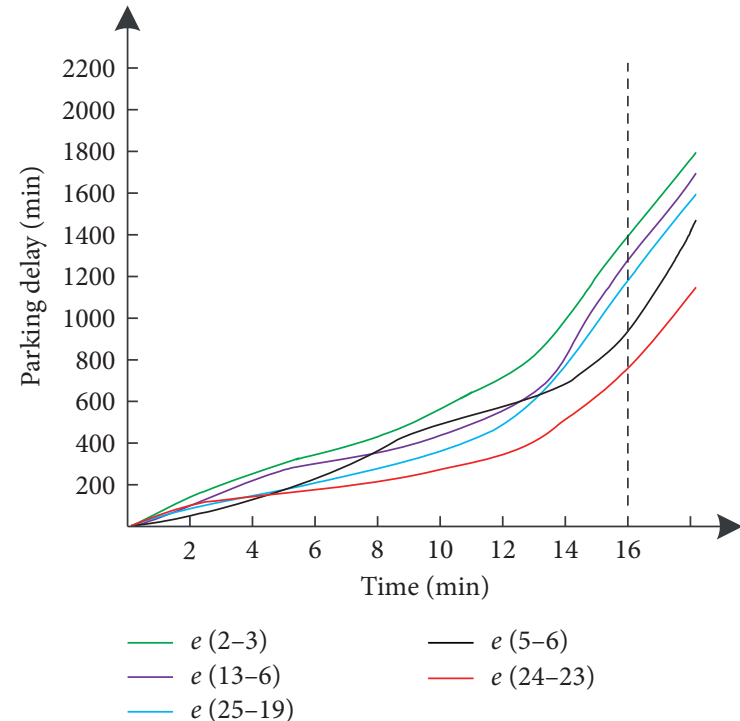

(a)

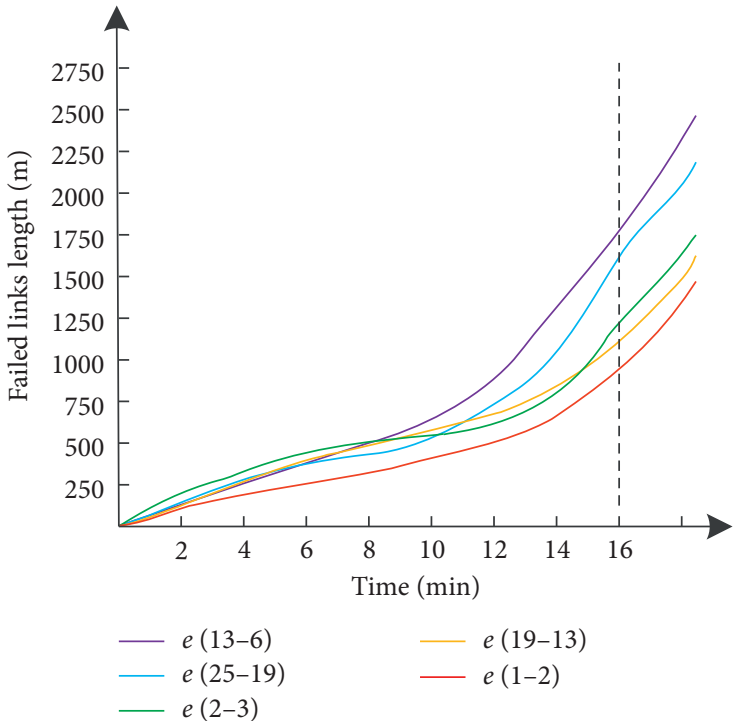

(b)

Figure 9: The criticality distribution of road links. (a) Description of results during the ordinary period. (b) Description of results during the peak period.

TABLE 2: Criticality ranking of load links during the ordinary period.

\begin{tabular}{lcc}
\hline Road link no. & Road link name & Rank \\
\hline$e(2-3)$ & North fourth ring road west auxiliary road/Caihefang road west to east & 1 \\
$e(13-6)$ & Zhongguancun street/North fourth ring road west auxiliary road-south to north & 2 \\
$e(25-19)$ & Zhongguancun street/Haidian street-south to north & 3 \\
$e(5-6)$ & North fourth ring road west auxiliary road/Zhongguancun street-west to east & 4 \\
$e(24-23)$ & Shanyuan road/Haidian east street-southwest to northeast & 5 \\
\hline
\end{tabular}


TABLE 3: Criticality ranking of load links during the peak period.

\begin{tabular}{lcc}
\hline Road link no. & Road link name & Rank \\
\hline$e(13-6)$ & Zhongguancun street/North fourth ring road west auxiliary road-south to north & 1 \\
$e(25-19)$ & Zhongguancun street/Haidian street-south to north & 2 \\
$e(2-3)$ & North fourth ring road west auxiliary road/Caihefang road west to east & 3 \\
$e(19-13)$ & Zhongguancun street/Haidian north street-south to north & 4 \\
$e(1-2)$ & North fourth ring road west/Suzhou street-west to east & 5 \\
\hline
\end{tabular}

weaker stability and larger proportion of vehicles in the nearby road network. This indicates that the identification results of this method not only consider the influence of road network topology on the criticality of road links but also take into account the traffic flow factors. Combined with the user's daily travel experience and real-time road condition data to analyze the identification results of this method, it is consistent with the traffic traveler's perception of the criticality of road links.

\section{Conclusions}

The main conclusions got from this paper are as follows.

(1) We proposed a road link cascade failure impact model, and the results of simulation experiments show that the accuracy of the total parking delay is $90.6 \%$ during the ordinary period, and the accuracy of failure road links length is $91.6 \%$ during the peak period, which indicates that the model can effectively evaluate the impact of dynamic traffic flow on road network after the failure of road links.

(2) The identification model of critical links we proposed considers the impact of cascading failures, and it can objectively reflect the dynamic impact on the road network after the failure of road links. This method identifies that arterial road links and bottleneck road links are the key to improve the stability of road networks, which can provide a basis for the congestion source management in urban road traffic and also provide a theoretical reference for the active control of traffic signals.

\section{Data Availability}

The data included in this paper are available without any restriction.

\section{Conflicts of Interest}

The authors declare that they have no conflicts of interest.

\section{Acknowledgments}

This research was funded by the National Key Research and Development Program of China (no. 2018YFB1601003), National Natural Science Foundation of China (no. 61673022), Natural Science Foundation of Beijing (no. 8172018), Science and Technology Program of Beijing Municipal Education Commission (no. KM202111417003), Talent Strengthening School Optimization Plan of Beijing
Union University (no. BPHR2020DZ04), and Beijing Shijingshan District Science and Technology Plan Project (no. 2020NCUT401053762034).

\section{References}

[1] M. A. P. Taylor, "Critical transport infrastructure in urban areas: impacts of traffic incidents assessed using accessibilitybased network vulnerability analysis," Growth and Change, vol. 39, no. 4, pp. 593-616, 2008.

[2] M. Stephen, Streets and Patterns: The Structure of Urban Geometry, Spon Press, London, UK, 2005.

[3] P. Timmerman, Vulnerability Resilience and Collapse of Society, Institute for Environmental Studies, Toronto, ON, Canada, 1981.

[4] K. Berdica, "An introduction to road vulnerability: what has been done, is done and should be done," Transport Policy, vol. 9, no. 2, pp. 117-127, 2002.

[5] E. Jenelius and L. G. Mattsson, "Developing a methodology for road network vulnerability analysis," Nectar Cluster, vol. 1, no. 1, pp. 1-9, 2006.

[6] G. Lleras-Echeverri and M. Sánchez-Silva, "Vulnerability analysis of highway networks, methodology and case study," Proceedings of the Institution of Civil Engineers-Transport, vol. 147, no. 4, pp. 223-230, 2001.

[7] M. Sánchez-Silva, M. Daniels, G. Lleras, and D. Patiño, “A transport network reliability model for the efficient assignment of resources," Transportation Research Part B: Methodological, vol. 39, no. 1, pp. 47-63, 2005.

[8] G. D’ ESTE, "Modelling network vulnerability at the level of the national strategic transport network," Journal of the Eastern Asia Society for Transportation Studies, vol. 4, no. 2, pp. 1-14, 2001.

[9] X. P. Zhang, Y. S. Li, G. Liu et al., "Evaluation method of importance for nodes in complex networks based on importance contribution," Complex Systems and Complexity Science, vol. 11, no. 3, pp. 26-32, 2014.

[10] Y. J. Li and X. Luo, “"Identification of urban road network's critical links set under emergency environment," Journal of Transportation Systems Engineering and Information Technology, vol. 18, no. 2, pp. 128-135, 2018.

[11] Y. F. Tu, C. Yang, and X. H. Chen, "Analysis of road network topology vulnerability and critical links," Journal of Tongji University, vol. 38, no. 3, pp. 364-367, 2010.

[12] M. O. Bell, B. L. Golden, and R. V. Vohra, "Finding the most vital areas in a network," Operations Research Letters Research Letters, vol. 8, no. 2, pp. 73-76, 1989.

[13] M. Girvan and M. Newman, "Community structure in social and biological networks," Proceedings of the National Academy of Sciences of USA, vol. 99, no. 12, pp. 7821-7826, 2001.

[14] Z. M. Lu and Y. P. Feng, "Critical nodes and links evaluation with multi-criteria based on entropy-weighted method," Journal of Information Hiding \& Multimedia Signal Processing, vol. 6, no. 6, pp. 1062-1076, 2015. 
[15] Y. Zhao, H. Li, and F. Wang, "Identifying key sections in urban road network based on k-shell," Systems Engineering, vol. 32, no. 5, pp. 105-110, 2014.

[16] H. Q. Song, J. Guo, and G. Liu, “Auto generalization approach and importance evaluation of urban roads based on complex networks," Engineering of Surveying \& Mapping, vol. 26, no. 1, pp. 8-12, 2017.

[17] D. M. Scott, D. C. Novak, L. Aultman-Hall, and F. Guo, "Network Robustness Index: a new method for identifying critical links and evaluating the performance of transportation networks," Journal of Transport Geography, vol. 14, no. 3, pp. 215-227, 2006.

[18] J. L. Sullivan, D. C. Novak, L. Aultman-Hall, and D. M. Scott, "Identifying critical road segments and measuring systemwide robustness in transportation networks with isolating links: a link-based capacity-reduction approach," Transportation Research Part A: Policy and Practice, vol. 44, no. 5, pp. 323-336, 2010.

[19] J. X. Zhang and Y. Jiang, "Criticality calculation of road links based on local network traffic flow redistribution," Journal of Transportation Systems Engineering and Information Technology, vol. 16, no. 1, pp. 105-110, 2016.

[20] A. W. Kuang and G. N. Lin, "Ranking of link importance considering route redundancy and travel efficiency," Journal of Changsha University of Science \& Technology, vol. 166, no. 2, pp. 1-7, 2019.

[21] Z. Y. Zhou, B. H. Mao, Q. Z. Gong et al., "Failure correlation analysis of urban road network," Journal of Transportation Systems Engineering and Information Technology, vol. 9, no. 2, pp. 110-114, 2009.

[22] R. L. Wu, H. Yang, and T. Liu, "Benefit evaluation index and methods for road intersection signal control," Journal of Nanjing Normal University, vol. 42, no. 2, pp. 37-43, 2019. 